\title{
Immune globulin subcutaneous, human - klhw $20 \%$ for primary humoral immunodeficiency: an open-label, Phase III study
}

\author{
John W Sleasman*,1, William R Lumry², Iftikhar Hussain ${ }^{3}$, H James Wedner ${ }^{4}$, James B \\ Harris $^{5}$, Kecia L Courtney ${ }^{6}$, Elsa Mondou ${ }^{6}$, Jiang Lin 6 \& Mark R Stein ${ }^{7}$ \\ ${ }^{1}$ Division of Allergy, Immunology, \& Pulmonary Medicine, Duke University School of Medicine; DUMC Box 2644, 203 Research \\ Drive, Room 133B MSRB 1, Durham, NC 27710, USA \\ ${ }^{2}$ Allergy \& Asthma Specialists; 10100 N. Central Expressway Suite 100 Dallas, TX 75231, USA \\ ${ }^{3}$ Vital Prospects Clinical Research Institute, PC, 7307 S. Yale Avenue, Tulsa, OK 74136, USA \\ ${ }^{4}$ Division of Allergy \& Immunology, Washington University in St. Louis, 4921 Parkview Place, Fl 8, Saint Louis, MO 63110, USA \\ ${ }^{5}$ Allergy \& Immunology, The South Bend Clinic Center for Research; 211 North Eddy St. South Bend, IN 46617, USA \\ ${ }^{6}$ Grifols Bioscience Research Group, Grifols, 4201 Research Commons, 79 TW Alexander Drive, Research Triangle Park, NC 27709, \\ USA \\ ${ }^{7}$ Good Samaritan Medical Center; 1309 N Flagler Dr, West Palm Beach, FL 33401, USA \\ *Author for correspondence: Tel.: +1 919681 2949; john.sleasman@duke.edu
}

\begin{abstract}
Aim: This prospective, Phase III study assessed the pharmacokinetics (PK), safety and tolerability of immune globulin subcutaneous, human - klhw $20 \%$ solution (IGSC-C $20 \%$ ) in participants with primary humoral immunodeficiency (PI), compared with immune globulin injection (human), 10\% caprylate/chromatography purified (IGIV-C 10\%). Patients \& methods: About 53 participants enrolled. Total 44 received IGIVC $10 \%$ in the run-in phase and then entered the IV phase (with an additional nine who were already receiving IGIV-C $10 \%$ and entered the IV phase directly) for steady-state IV PK assessments. Total 49 entered the SC phase (weekly doses of IGSC-C $20 \%$ for $\sim 24$ weeks). The PK profiles of IGIVC $10 \%$ and IGSC-C $20 \%$ and their safety and tolerability parameters were compared. Results: At a dose adjustment factor of 1.37 , IGSC-C $20 \%$ provided comparable (noninferior and bioequivalent) overall total immunoglobulin G exposure to IGIV-C $10 \%$ over an equal time interval. About 33 participants reported 79 adverse events during run-in + IV phases; 41 participants reported 141 adverse events during the SC phase, with most being local infusion site reactions. The majority of infusion site reactions were mild to moderate in severity. Conclusion: IGSC-C $20 \%$ was bioequivalent to IGIVC $10 \%$ and was well tolerated, with a safety profile comparable with IGIV-C 10\%, in this study. Trial registration: ClinicalTrials.gov identifier: NCT02604810

Lay abstract: This clinical trial studied a new US FDA-approved product, immune globulin subcutaneous, human - klhw $20 \%$ solution (IGSC-C $20 \%$ ), to treat primary humoral immunodeficiency (PI), a group of inherited disorders in which part of the immune system is missing or malfunctioning. Patients with PI may be more susceptible to infections. This new product is a liquid medicine that is injected into the fat under the skin and contains immunoglobulin $\mathrm{G}$ antibodies, which may be used to protect the body against infection. Of the 53 enrolled participants, 42 (79\%) completed the study. Results from this study showed that IGSC-C $20 \%$ acted similarly in the body as immune globulin injection (human), 10\% caprylate/chromatography purified (IGIV-C $10 \%$ ), which is given in the vein and has been used for PI since 2003 . IGSC-C $20 \%$ was well tolerated and safe in adults and children, as young as 2 years old, in this study.
\end{abstract}

First draft submitted: 6 September 2019; Accepted for publication: 30 September 2019; Published online: 17 October 2019

Keywords: $20 \%$ immunoglobulin • bacterial • immunoglobulin replacement therapy • pharmacokinetics $\bullet$ primary immunodeficiency disease $\bullet$ subcutaneous administration • subcutaneous immunoglobulin • Xembify 
As of February 2017, the International Union of Immunological Societies reported 344 different inborn errors of immunity underlying 354 distinct disorders that compose primary immunodeficiency diseases, also known globally by abbreviations PID or PIDD [1]. And with the rise of next-generation gene sequencing and increased recognition of immune dysregulation diseases (i.e., autoinflammatory disorders and interferonopathies), this disease landscape is evolving rapidly, with phenotypes as diverse as infection, malignancy, allergy, autoimmunity and autoinflammation [1]. Primary humoral immunodeficiency (PI) consists of a heterogeneous group of genetic disorders comprising the majority of primary immunodeficiencies. Patients with impaired humoral immunity are highly susceptible to a wide range of infections, most commonly bacterial infections [2]. PI includes, but is not limited to, congenital agammaglobulinemia, common variable immunodeficiency, $x$-linked agammaglobulinemia, Wiskott-Aldrich syndrome and severe combined immunodeficiencies. Patients with PI are particularly susceptible to serious bacterial infections of the respiratory tract as well as other infections [2,3]. In the USA one, approximately one in 2000 children and one in 1200 persons (including adults and children) have primary immunodeficiencies, and those needing antibody replacement receive regular infusions of immune globulin (Ig) as immune prophylaxis to prevent recurrent infections $[4,5]$. Human Ig used for the treatment of PI consists of purified human immunoglobulin $\mathrm{G}(\mathrm{IgG})$ obtained from multiple plasma donors and has been well established as an efficacious treatment of antibody deficiency disorders [6,7]. Administration of Ig in a variety of IgG preparations can be carried out via intramuscular, intravenous (IV) or subcutaneous (SC) route.

Over the past two decades, Ig given subcutaneously (IGSC) has become an increasingly popular route for IgG replacement therapy [8]. Seven IGSC products, with IgG protein concentrations ranging from 10 to 20\%, are currently approved by the FDA to treat PI by SC administration [9-14]. However, only two of the seven FDAapproved IGSC products have $20 \% \mathrm{IgG}$ protein concentration [10,11], and not all patients have access to these $20 \%$ IgG formulations. The higher protein concentration of $20 \%$ products reduces infusion volume and allows shorter infusion time, which makes them easier to administer subcutaneously. IGSC allows the flexibility of patient selfadministration and provides another treatment option for physicians and patients to treat PI without intravenous infusions [12].

We report results on the pharmacokinetics (PK), safety and tolerability of this new IGSC-C 20\% treatment option in patients with PI from a recently completed, prospective, multicenter and Phase III clinical study (NCT02604810). The investigational product in this study was the new Ig subcutaneous, human - klhw 20\% solution (IGSC-C 20\%) approved by the FDA in July 2019 for the treatment of PI in patients 2 years of age and older [13]. The second study drug, Ig injection (human), 10\% caprylate/chromatography purified (IGIV-C 10\%) [14], was employed in this trial to standardize IV Ig administration during the initial run-in phase and IV phase.

\section{Patients \& methods}

This study was sponsored by Grifols Therapeutics LLC (Research Triangle Park, NC, USA) and conducted by Novella (NC, USA). The central laboratory was Covance (Indianapolis, IN, USA). Pharmacokinetic analyses were done by Nuventra Pharma Sciences (NC, USA).

\section{Study participants}

All participants screened during the screening phase (lasting up to 28 days) had a confirmed diagnosis of primary immunodeficiency, which included humoral and combined deficiencies (e.g., hyper IgM syndrome and severe combined immunodeficiency post-transplantation), as defined by the Pan-American Group for Immunodeficiency and the European Society for Immunodeficiencies [2], and were clinically stable on replacement immunoglobulin with a screening IgG trough $\geq 500 \mathrm{mg} / \mathrm{dl}$. A complete list of inclusion and exclusion criteria is in Supplementary Table 1. About 50 participants were planned for enrollment to provide 30 completing adults and $12-18$ completing children, with a target of four to six children for each age group ( $\geq 2-5,>5-12$ and $>12-16$ years). The planned minimum enrollment of 42 completing participants provided $\geq 90 \%$ power to demonstrate that the area under the curve (AUC) for total IgG for IGSC-C $20 \%$ was noninferior to that achieved by IGIV-C 10\%, assuming the intrasubject coefficient of variation was no greater than $30 \%$. 


\section{Study product}

The study drug IGSC-C 20\% (XEMBIFY ${ }^{\circledR}$ [Ig subcutaneous, human - klhw] 20\% solution) [13] is a new product manufactured with the same caprylate/chromatography purification process and stabilizer (glycine) as IGIV-C $10 \%$ (Gamunex ${ }^{\circledR}-\mathrm{C}$ [Ig injection (human), 10\% caprylate/chromatography purified] [14]. Both IGSC-C 20\% and IGIV-C 10\% are manufactured by Grifols Therapeutics LLC (Research Triangle Park, NC, USA) from large pools of human plasma by a combination of cold ethanol fractionation, caprylate precipitation and filtration, and anionexchange chromatography. IGSC-C $20 \%$ is a ready-to-use sterile, nonpyrogenic solution of high purity ( $\geq 98 \%)$ human $\mathrm{IgG}$ protein for subcutaneous infusion, with a final formulation of $20 \%(\mathrm{w} / \mathrm{v})$ protein concentration, a $\mathrm{pH}$ of 4.1-4.8, trace amounts of IgA and IgM, and an osmolality of about $340 \mathrm{mOsmol} / \mathrm{kg}$.

\section{Study design}

This prospective, multicenter, open-label, single-sequence and Phase III study (Figure 1) took place between 4 January 2016 and 14 December 2017. The primary PK objective of this study was to determine a weekly dose of IGSC-C 20\% that produced steady-state AUC of total IgG that was noninferior to that of the regularly administered dose of IGIV-C $10 \%$ in participants with PI. The secondary PK objective was to determine whether IGSC-C 20\% replacement therapy maintained mean steady-state trough of total IgG levels that were comparable with IGIV-C $10 \%$. The safety objective was to assess the safety and tolerability of IGSC-C $20 \%$ as an IgG replacement therapy in participants with PI. This study also evaluated several exploratory objectives, including trough levels of IgG subclasses, maximum concentration $\left(\mathrm{C}_{\max }\right)$ and time to reach maximum concentration $\left(\mathrm{t}_{\max }\right)$ at steady state, rate of serious bacterial infections, all infections of any kind as determined by the investigator, validated infections; number of days on antibiotics, use of prophylactic antibiotics, number of hospitalizations due to infection, and number of days of work/school/daily activities missed due to infections and their treatment.

The study consisted of a screening phase, a run-in phase, an IV phase, a SC phase and an end of study/early termination visit (Figure 1). The run-in phase was included to allow participants, who were not receiving IGIV-C $10 \%$ at $300-800 \mathrm{mg} / \mathrm{kg}$ per infusion every $3-4$ weeks for $\geq 3$ consecutive months before screening, to reach an approximate steady-state condition prior to PK profiling in the IV phase. The IV phase served as the reference (control) study phase in that the primary objective of noninferiority for IgG exposure under approximate steadystate conditions would be determined by comparing the AUC for SC dosing $\left(\mathrm{AUC}_{\mathrm{sc}}\right.$ ) of IGSC-C $20 \%$ against that from IV dosing $\left(\mathrm{AUC}_{\mathrm{IV}}\right.$ ) of IGIV-C 10\%. Participants who went through the IV phase received the same dose of IGIV-C $10 \%$ as had been administered when they were in the run-in period. During the SC phase, participants received weekly SC doses of IGSC-C $20 \%$ for 24 weeks. The dosing regimen for IGSC-C $20 \%$ was based on literature data of the bioavailability of SC administered IgG and prior experience with IGIV-C $10 \%$ given subcutaneously [15-19]. The weekly IGSC-C 20\% dosing regimen (1.37-times the IV dose of IGIV-C 10\% divided by either 3 or 4 weeks depending on the dosing interval) was predicted to provide a noninferior steady-state IgG AUC to that of the prior IGIV-C 10\% dose based on established bioequivalence criteria. The PK profiles were assessed for all participants. Participants aged 2-5 years old had an abbreviated sampling schedule for PK profiles.

Participants and investigators were allowed to tailor the number of injection sites, infusion rate and the specific times of the day for the SC infusion. Up to eight infusion sites ( $\geq 5 \mathrm{~cm}$ apart) per infusion were allowed, and participants were allowed to use the same anatomical area or rotated anatomical areas for SC infusions throughout the study. The target infusion rate was $\leq 25 \mathrm{ml} / \mathrm{h} /$ site, depending on tolerability. Once the target infusion rate was achieved, it was not changed in the middle of an infusion unless the subject experienced tolerability issues at that infusion rate. In the event, the subject was not able to tolerate a set infusion rate, the rate could be decreased for better tolerability. Conversely, if the target infusion rate was well tolerated during two infusions, an increase of $20 \%$ in infusion rate and volume per site was allowed at the discretion of the investigator.

An interim PK analysis was conducted on eight participants (aged 27-72 years) to confirm that a SC dose adjustment factor (DAF) of 1.37 was adequate. The DAF was deemed acceptable if the ratio of geometric least squares mean (GLSM) for the area under the concentration-time curve from 0 to 7 days following SC infusion ( $\mathrm{AUC}_{0-7, \mathrm{SC}}$ ) versus the adjusted area under the concentration-time curve from 0 to 7 days following IV infusion $\left(\mathrm{AUC}_{0-7, \mathrm{IV}}\right)$ was $\geq 0.9$. Data from this interim analysis yielded a ratio of 0.95 , mean trough $\mathrm{IgG}$ values $\geq 757 \mathrm{mg} / \mathrm{dl}$ for individual participants receiving IGSC-C $20 \%$, and an average mean trough IgG level across participants of $1169 \mathrm{mg} / \mathrm{dl}$. Thus, the DAF of 1.37 was acceptable, and no modification was warranted. 


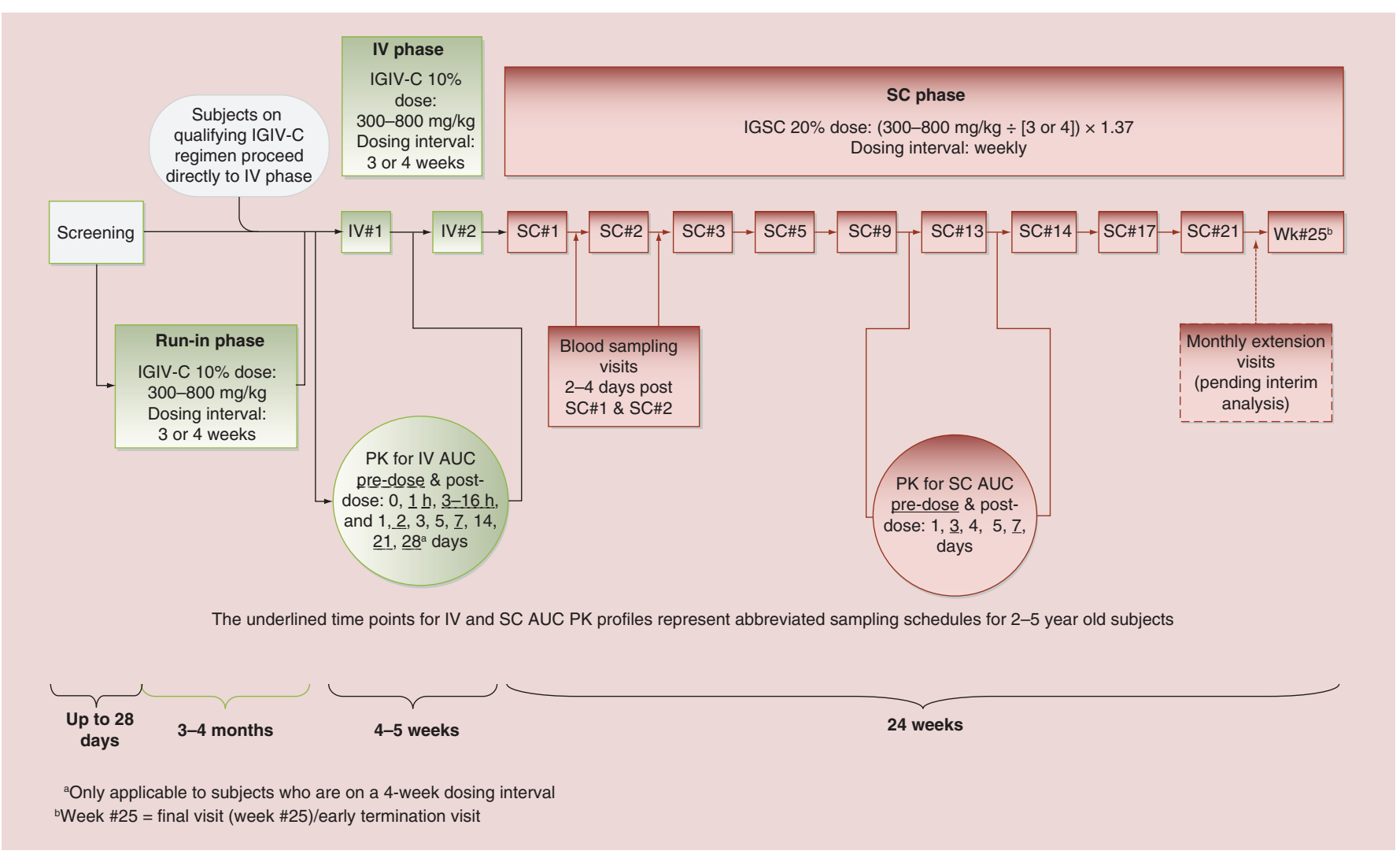

Figure 1. Study design. Study entry point was determined at screening based on the most recent lgG treatment history of each subject. During the screening phase of up to 28 days following the first assessment of subject eligibility, participants were categorized into three groups. Group 1: Participants who were receiving a stable ( $\geq 3$ months) dose of IV IGIV-C $10 \%$ between 300 and $800 \mathrm{mg} / \mathrm{kg}$ per infusion every 3 or 4 weeks did not need to enter the run-in phase and proceeded directly to the IV phase; these participants had their IV\# 1 visit scheduled to coincide with the date for their next IV infusion according to their regular dosing interval $(\tau)$ and came to the clinic for this visit. Group 2: Participants who had been receiving a different commercially available IGIV therapy other than IGIV-C $10 \%$ at a dose between 300 and $800 \mathrm{mg} / \mathrm{kg}$ per infusion every 3 or 4 weeks were required to receive IGIV-C $10 \%$ intravenously (at an equivalent dose and dosing interval as in their previous IGIV therapy) for a total duration of a 3-month run-in phase; participants receiving infusions every 3 weeks had four run-in visits and participants receiving infusions every 4 weeks had three run-in visits. Group 3: Participants were required to participate in a 4-month run-in phase if they had been receiving SCIG prior to screening, or if they had been receiving IGIV therapy other than IGIV-C $10 \%$ but had not been on a stable dose for the 3 months prior to screening OR the dose was not between 300 and $800 \mathrm{mg} / \mathrm{kg}$ OR who received IGIV infusions that were not administered at a frequency of every 3 or 4 weeks. Participants meeting any of the criteria for Group 3 were scheduled to begin receiving IGIV C $10 \%$ intravenously at a dose of 300-800 mg/kg every 3 to 4 weeks (dose and interval were determined based on the investigator's clinical judgment) for a period of up to 4 months prior to the IV\# 1 visit. Participants receiving infusions every 3 weeks had five run-in visits and participants receiving infusions every 4 weeks had four run-in visits. The AUC from the IV phase of the study was calculated based on trough-to-trough measurements around the IV\# 1 infusion. Blood samples were taken immediately prior to the infusion and then at specified times (for 3-4 weeks depending on dosing interval); the last blood-draw for AUC for IV dosing (AUCIV) occurring just before the IV\#2 infusion. The SC phase occurred 1 week after the second IV dose (IV\#2). The IGSC-C $20 \%$ dose was determined using an initial IV to SC dose adjustment factor of 1.37. To determine a SC DAF, a comparison of the IV PK profiles and SC PK profiles in the first eight participants (age $\geq 12$ to 75 years) was performed through an interim PK analysis. Since a DAF of 1.37 was deemed acceptable (SC dose of IGSC-C $20 \%$ was noninferior to the subject's IV dose), all participants continued and completed treatment and assessments through Week \#25 (no further monthly extension visits). PK profiles were assessed in all participants. Participants aged 2-5 years old were assessed using an abbreviated sampling schedule (underlined time points) for PK profiles. The first three weekly SC doses were administered in the clinic to ensure the subject utilized the proper self-administer techniques. SC infusions 5, 9, 17 and 21 were performed in the clinic to obtain total IgG trough levels before beginning the infusion. The remaining weekly SC infusions were self-administered at home. After 12 weeks of weekly SC therapy, determination of PK profiles for total IgG during the SC phase began just prior to the 13th SC infusion with the last sample collected immediately prior to the 14th SC infusion (7 days total).

AUC: Area under the concentration versus time curve; DAF: Dose adjustment factor; IGIV-C 10\%: Immune globulin injection (human), $10 \%$ caprylate/chromatography purified (Grifols); IGSC-C 20\%: Immune globulin subcutaneouss (human), 20\% caprylate/chromatography purified (Grifols); IGIVI: Intravenous immune globulin (generic terminology); IV, intravenous; PK: Pharmacokinetic/pharmacokinetics; SC: Subcutaneous; SCIG: Subcutaneously delivered immune globulin or subcutaneous immunoglobulin (generic terminology). 
All participants received study drug treatment in the same order (i.e., IV infusion of IGIV-C $10 \%$ followed by SC infusion of IGSC-C 20\%). The duration of the IGSC-C 20\% phase was approximately 24 weeks for all participants.

\section{Statistical analyses}

Descriptive statistics were calculated as appropriate for the type of end points (continuous/quantitative or categorical/qualitative). Geometric mean and $90 \% \mathrm{CI}$ were calculated for PK parameters (except $\mathrm{t}_{\max }$ ). The safety population included participants who received any amount of study drugs (IGIV-C 10\% and/or IGSC-C 20\%). The IgG population consisted of participants who received study drugs and had any total IgG concentration data. The PK population consisted of all participants who received study drugs and had sufficient, valid data to calculate steady-state $\mathrm{AUC}_{0-\tau, \mathrm{SC}}$ or $\mathrm{AUC}_{0-\tau, \mathrm{IV}}$, which were analyzed by analysis of variance with a mixed-effect model. PK parameters were calculated using standard noncompartmental methods. For infection-related end points, the rate per person per year and associated 95\% CI were calculated using a generalized linear model for Poisson regression.

\section{Results}

\section{Participants}

Of the 61 participants screened by 20 study centers from the USA and Canada, 53 entered the study (safety population and IgG population), and 50 were evaluable for the PK analysis population (Supplementary Figure 1). Three participants were excluded from the PK population due to withdrawal from the run-in phase $(n=1)$ and the lack of sufficient total IgG data for AUC calculations $(n=2)$. About 49 participants completed the IV phase and entered the SC phase, receiving weekly IGSC-C 20\% for approximately 24 weeks, with PK profiling at SC week \#13-14. Of the 53 who entered the study, 42 participants (79.2\%) completed the study, and 11 participants (20.8\%) discontinued the study prematurely due to adverse events (AEs) $(n=5)$, withdrawal by subject $(\mathrm{n}=4)$, lost to follow-up $(\mathrm{n}=1)$ and other reason $(\mathrm{n}=1)$. Pediatric enrollment occurred as per protocol; only two participants were enrolled in the $\geq 2$ to $\leq 5$ year age group despite significant efforts to identify participants for this age category. Table 1 shows the demographics and baseline characteristics for the safety population. The average (standard deviation [SD]) time from diagnosis of PI to study entry was 10.3 (10.7) years (Table 2); there were no major differences in the history of PI and IgG treatment across age groups. The most frequently associated medical diagnoses were asthma $(37.7 \% ; 20$ of 53) and allergic rhinitis $(35.8 \% ; 19$ of 53$)$.

\section{Pharmacokinetic parameters}

Primary PK end points were analyzed for three versions of the PK population: the full PK population ( $\mathrm{n}=49$ in IV phase and $n=39$ in SC phase), the PK population with the original eight participants used in the interim analysis excluded, and only participants in the PK population with sufficient and valid serial PK profiles that allowed the calculation of the AUC parameter in both the IV and SC phases. At a DAF of 1.37, the GLSM ratio (SC/IV) for the primary PK end point of steady state $\mathrm{AUC}_{0-7}$ days ranged from 1.04 (90\% CI: $\left.1.00-1.07\right)$ to 1.06 (90\% CI: 1.03-1.10), demonstrating that SC administration of IGSC-C $20 \%$ is noninferior and bioequivalent to regularly administered IV dose of IGIV-C 10\% (Figure 2A), and there was no difference between age groups (data not shown).

Results from the IV phase $(\mathrm{n}=49)$, including participants who received IV doses of IGIV-C $10 \%$ every 3 weeks $(n=6)$ and every 4 weeks $(n=43)$, showed the average $(S D)$ predose IgG trough (i.e., prior to infusion IV\#1) was $964(242) \mathrm{mg} / \mathrm{dl}$. At the completion of the first IV infusion, the average total IgG concentration rose to 2075 (396) $\mathrm{mg} / \mathrm{dl}$, followed by a decrease to $913(192) \mathrm{mg} / \mathrm{dl}$ at the end of the 4-week dosing interval. The average predose IgG trough prior to SC\#13 infusion of IGSC-C 20\% was 1253 (279) $\mathrm{mg} / \mathrm{dl}$, and all participants maintained IgG levels $>580 \mathrm{mg} / \mathrm{dl}$ during the SC phase. Unlike the IV phase, which exhibited a clear peak in IgG concentration followed by an elimination phase, average total IgG concentrations following SC administration remained relatively stable, with only small fluctuations from preinfusion through 7 days post-SC infusion. The peak IgG level occurred at an average of $76 \mathrm{~h}$ after IGSC-C 20\% infusion. Average total IgG concentrations over 7 days of the PK profiling during the SC\#13 dosing interval ranged from the lowest of $1263(294) \mathrm{mg} / \mathrm{dl}$ to the highest of $1358(279) \mathrm{mg} / \mathrm{dl}$.

\section{Infection rates}

The number of participants with SBIs, validated infections and infections of any kind were similar between the IV and SC phases. Table 3 shows infection results for the combined run-in + IV and SC phases. No SBI was reported 
Table 1. Demographics and baseline characteristics (safety population).

\begin{tabular}{|c|c|c|c|}
\hline \multirow[t]{2}{*}{ Demographic variables } & & \multicolumn{2}{|c|}{ Total $(n=53)$} \\
\hline & & Mean \pm SD or $n(\%)$ & Median (min-max) \\
\hline \multicolumn{2}{|l|}{ Age, years } & $36.8 \pm 21.36$ & $39.0(2-72)$ \\
\hline \multirow[t]{4}{*}{ Age category, years } & $\geq 2$ to $\leq 5$ & $2(3.8)$ & - \\
\hline & $>5$ to $\leq 12$ & $7(13.2)$ & - \\
\hline & $>12$ to $\leq 16$ & $6(11.3)$ & - \\
\hline & $>16$ & $38(71.7)$ & - \\
\hline \multirow[t]{2}{*}{ Sex } & Male & $27(50.9)$ & - \\
\hline & Female & $26(49.1)$ & - \\
\hline \multirow[t]{2}{*}{ Ethnicity } & Hispanic or Latino & $5(9.4)$ & - \\
\hline & Non-Hispanic or -Latino & $48(90.6)$ & - \\
\hline \multicolumn{2}{|l|}{ Weight, kg } & $68.4 \pm 25.5$ & $66.5(16.7-123.8)$ \\
\hline \multicolumn{2}{|l|}{ Baseline total lgG ${ }^{\dagger} \mathrm{mg} / \mathrm{dl}(\mathrm{n}=52)$} & $934.7 \pm 230.8$ & $917.5(545-1740)$ \\
\hline \multirow[t]{3}{*}{ Subject entry status } & $\begin{array}{l}\text { Subject entered in 3-month run-in } \\
\text { phase }\end{array}$ & $19(35.8)$ & - \\
\hline & $\begin{array}{l}\text { Subject entered in 4-month run-in } \\
\text { phase }\end{array}$ & $25(47.2)$ & - \\
\hline & Subject entered directly into IV phase & $9(17.0)$ & - \\
\hline \multirow{2}{*}{$\begin{array}{l}\text { Frequency of subject IV dose at entry } \\
\text { (run-in or IV phase) }\end{array}$} & Every 3 weeks & $6(11.3)$ & - \\
\hline & Every 4 weeks & $47(88.7)$ & - \\
\hline Assigned IV dose at entry, ${ }^{\ddagger} \mathrm{mg} / \mathrm{kg}$ & Every 3 weeks & $393.0 \pm 64.8$ & $387.5(324.0-483.0)$ \\
\hline
\end{tabular}

during the run-in phase. During the IV phase, two SBIs (pneumonia and sepsis) occurred in one participant (1.9\%, 1/52). During the SC phase, one SBI (due to an animal bite leading to sepsis and cellulitis) was reported in one participant $(2.0 \%, 1 / 49)$. All SBIs were treated and resolved. The rate of SBIs was 0.049 (95\% CI: 0.020-0.098; upper 99\% confidence limit: 0.110) events per subject-year during IGSC-C 20\% treatment and 0.120 (95\% CI: 0.051-0.232; upper 99\% confidence limit: 0.259) in the combined run-in + IV phase. These annual rates were lower than 1.0 SBI/year, the threshold specified as providing substantial evidence of efficacy while receiving IgG replacement therapy $[17,18]$.

The incidences of validated infections were similar between the combined run-in + IV phases and SC phase. In the combined run-in + IV phases, $15.1 \%$ (eight of 53) of participants had $\geq 1$ validated infection, and in the SC phase, $16.3 \%$ (eight of 49 ) of participants had $\geq 1$ validated infection. The rate of validated infections per person per year was comparable between the IV phases and SC phase: combined run-in + IV of $0.658(95 \%$ CI: $0.378-1.051)$ and SC phase of 0.493 (95\% CI: $0.273-0.809)$. The validated infections that occurred in $\geq 2$ participants in the combined run-in + IV phases were urinary tract infection and in the SC phase were cellulitis, pharyngitis streptococcal and urinary tract infection. Infections of any kind occurred in 50.9\% (27/53) and 53.1\% (26/49) of participants during the run-in + IV and the SC phases, respectively. 
Table 2. Summary of primary immunodeficiency and immunoglobulin G treatment history (safety population). Total $(n=53)$

Time since primary immunodeficiency diagnosis, years

\begin{tabular}{ll}
\hline- Mean \pm SD & $10.3 \pm 10.7$ \\
\hline- Median & 6.7 \\
\hline- Min-max & $0.3-41.1$
\end{tabular}

Type of primary immunodeficiency, $\mathrm{n}(\%)$

- Common variable immunodeficiency

41 (77.4)

- X-linked agammaglobulinemia

- Hyper IgM immunodeficiency syndrome

5 (9.4)

- Primary hypogammaglobulinemia

2 (3.8)

- Severe combined immunodeficiency (post-transplantation)

$2(3.8)^{\S}$

2 (3.8)

- Autosomal recessive agammaglobulinemia

1 (1.9)

Frequency of IgG treatment $†$ by route of administration for past 12 months, $\mathrm{n}$ (\%)

- IV administration:

- Every 3 weeks

6 (11.3)

- Every 4 weeks

$29(54.7)$

- SC administration:

- Every week

15 (28.3)

- Every 2 weeks

5 (9.4)

- Every 4 weeks $\ddagger$

$5(9.4)$

${ }^{\dagger}$ Ig treatments are not mutually exclusive.

$¥$ All SC and every-4-week IgG treatments were HYQVIA (immune globulin infusion 10\% [Human] with recombinant human hyaluronidase) solution for subcutaneous administration (Baxalta, MA, USA) [16].

$\S$ Both patients with primary hypogammaglobulinemia were children and were diagnosed with PI for over 2.5 years prior to study participation, and were maintained on SC immunoglobulin replacement prior to the study in order to provide adequate total IgG trough levels.

IgG: Immunoglobulin G: IV: Intravenous; SC: Subcutaneous; SD: Standard deviation.

Table 3. Summary of infections and associated events.

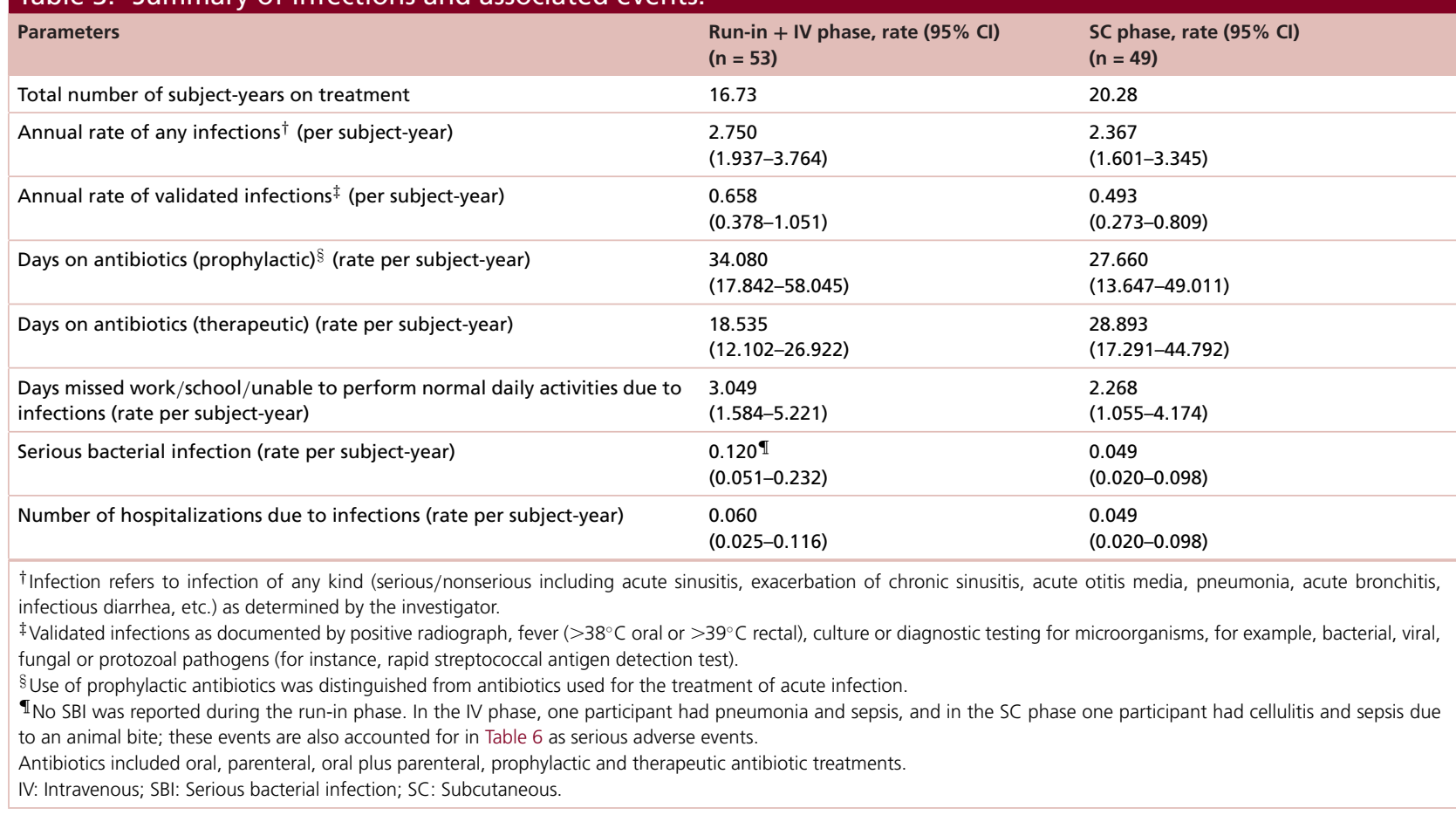




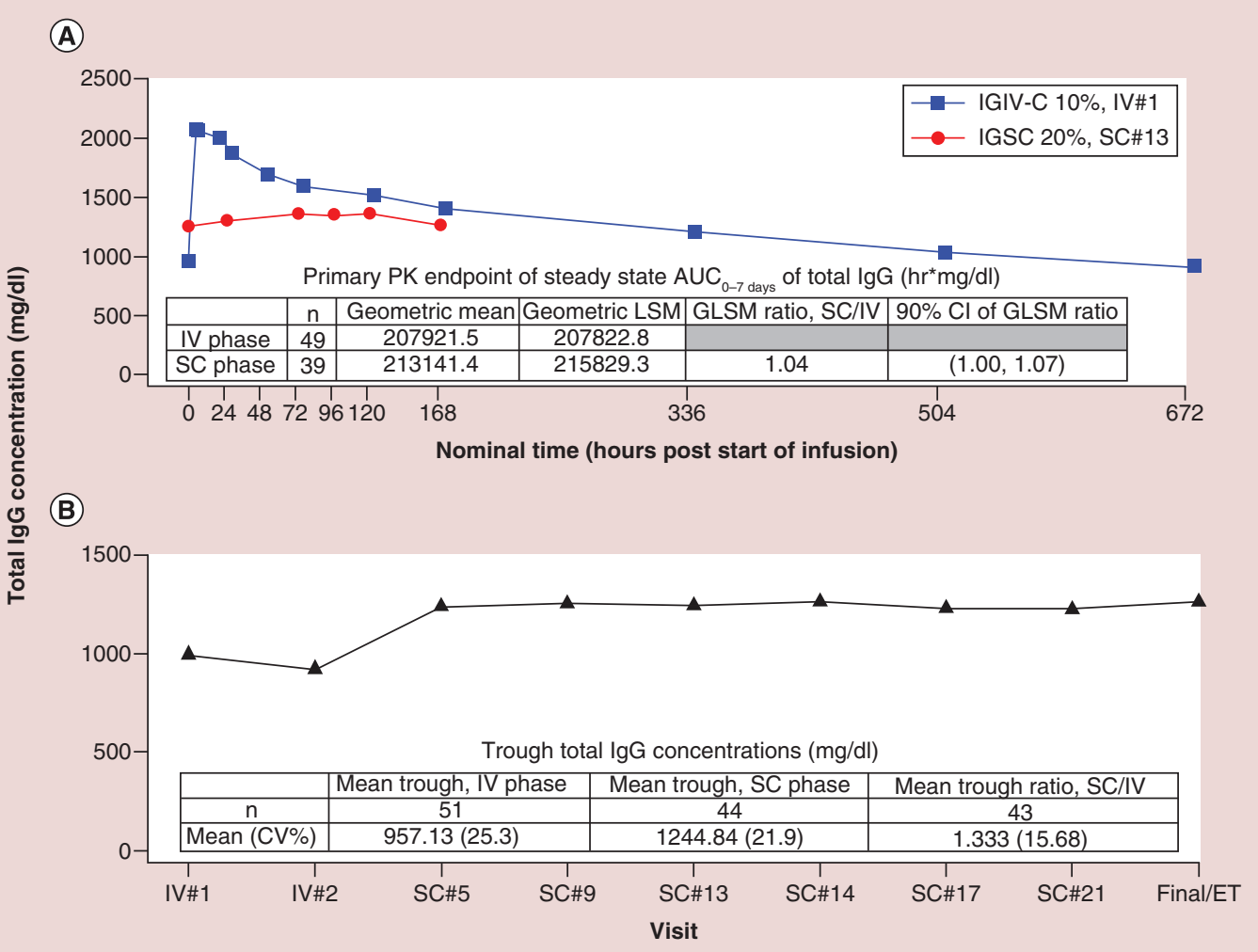

Figure 2. Mean total Immunoglobulin G concentrations (mg/dl). (A) Serial total lgG concentrations following IGIV-C $10 \%$ administration at IV\#1 and IGSC-C $20 \%$ administration at SC\#13. Inset table shows the statistical analysis results on the primary PK end point of steady state $A_{U C}$ 0-7 days of total IgG using the PK population. GLSMs, GLSM ratio and $90 \% \mathrm{Cl}$ of GLSM ratio are determined from a mixed-effect model with the log-transformed $\mathrm{AUC}_{0-7}$ days as the response variable, study phase as a fixed effect and subject as a random effect. $A U C_{0-21}$ days or $A U C_{0-28}$ days in the IV phase was standardized to $\mathrm{AUC}_{0-7}$ days by dividing it by 3 or 4 , depending on the IV dosing schedule. (B) Trough (predose) total IgG concentrations. Inset table shows the summary statistics using the IgG population. Mean trough in the IV phase is calculated as the average of the trough concentrations at the IV\#1 and IV\#2 visits. Mean trough in the SC phase is calculated as the average of the trough concentrations at the SC\#13, SC\#14, SC\#17 and SC\#21 visits. AUC: Area under the curve; CV: Coefficients of variation; IGIV-C 10\%: Immune globulin injection (human), 10\% caprylate/chromatography purified (Grifols); IGSC-C 20\%: Immune globulin subcutaneous (human), 20\% caprylate/chromatography purified (Grifols); GLSM: Geometric LSM; IV: Intravenous; LSM: Least-squares mean; PK: Pharmacokinetic; SC: Subcutaneous.

\section{Trough total IgG, IgG subclasses \& antibody titers}

Trough concentrations of total IgG are summarized in Figure 2B, along with the mean trough total IgG concentration during the IV and SC phases. The average (SD) of the steady-state mean trough concentrations of total IgG over all participants during the IV phase was approximately 957 (242) mg/dl. All individual trough concentrations, except for one subject, were above the commonly accepted protective trough concentration of $500 \mathrm{mg} / \mathrm{dl}$ [19]. There was a relatively small range of variation, with mean trough coefficients of variation (CV\%) of $25.3 \%$, which included a trend toward slightly higher trough values for the six participants with a 3-week dosing interval. The trough IgG concentration in the SC phase was consistent across the four visits, with CV\% ranging from 23 to $24 \%$. The average (SD) of the mean SC trough IgG concentrations over all participants was approximately 1245 (272) $\mathrm{mg} / \mathrm{dl}$ and was generally higher than the average of the mean IV trough IgG concentrations, with an average mean trough ratio (SC:IV) of 1.333. All individual trough concentrations in the SC phase were also above the accepted $500 \mathrm{mg} / \mathrm{dl}$ therapeutic threshold [20].

The results for $\operatorname{IgG}$ subclasses (IgG1, IgG2, IgG 3 and IgG4) in the two study phases were consistent with total IgG trough levels by visit (Supplementary Table 2). Trough IgG subclasses reached a steady state by at least $\mathrm{SC}$ week 


\begin{tabular}{|c|c|c|c|c|c|c|}
\hline Pathogen type & Statistic & \multicolumn{2}{|c|}{ IV phase } & \multicolumn{2}{|c|}{ SC phase } & $\begin{array}{l}\text { Final visit/early } \\
\text { termination visit }\end{array}$ \\
\hline \multirow{3}{*}{$\begin{array}{l}\text { Hemophilus } \\
\text { influenzae }(\mu \mathrm{g} / \mathrm{ml})\end{array}$} & $\mathrm{n}$ & 49 & 49 & 43 & 41 & 49 \\
\hline & Mean \pm SD & $5.4 \pm 9.5$ & $5.0 \pm 8.8$ & $6.6 \pm 9.1$ & $6.4 \pm 9.7$ & $8.5 \pm 14.5$ \\
\hline & Min-max & $0.4-64.0$ & $0.5-60.8$ & $0-60.9$ & $1.1-63.9$ & $0.9-89.3$ \\
\hline $\begin{array}{l}\text { Clostridium tetani } \\
(\mathrm{IU} / \mathrm{ml})\end{array}$ & Min-max & $1.2-10.5$ & $0.9-16.1$ & $2.1-14.7$ & $2.4-13.0$ & $0.8-17.4$ \\
\hline \multirow[t]{3}{*}{ Measles $^{\dagger}(\mathrm{IU} / \mathrm{ml})$} & $\mathrm{n}$ & - & 51 & - & - & 47 \\
\hline & Mean \pm SD & - & $1.5 \pm 1.2$ & - & - & $1.6 \pm 0.9$ \\
\hline & Min-max & - & $0.3-8.0$ & - & - & $0.4-6.3$ \\
\hline
\end{tabular}

†Trough samples for measles antibody titer were collected at IV\#2 (before the first SC infusion of IGSC-C 20\%) and at SC week \#52, and then measured by functional assay. IgG: Immunoglobulin G; IV: Intravenous; SC: Subcutaneous; SD: Standard deviation.

\#9, remained proportionally similar in both study phases, and were also generally consistent across the different age groups (data not shown).

The trough levels of antibodies for Hemophilus influenzae, Clostridium tetani and measles are shown in Table 4. Data for Streptococcus pneumoniae subtypes are shown in Supplementary Table 3. For H. influenzae, C. tetani and $S$. pneumoniae, serum trough concentrations were generally consistent across all age groups (data not shown). After switching from IV to SC administration, the average trough levels of these antibodies increased, and the increases remained through the final study visit. The trough concentrations of measles antibody were comparable between the IV and SC phases (Table 4), except for the $\geq 2$ to $\leq 5$ year age group $(n=2)$ which had increased from a mean of $0.475 \mathrm{IU} / \mathrm{ml}$ in the IV phase to $1.120 \mathrm{IU} / \mathrm{ml}$ in the SC phase. All measles antibody titers were protective (i.e., minimum range in excess of $0.24 \mathrm{IU} / \mathrm{ml}$ ) [21,22].

\section{Subcutaneous administration characteristics}

Table 5 summarizes the administration characteristics of IGSC-C 20\% in this study. Most SC infusions were conducted using either four infusion sites (56.2\%, 592 of 1053 infusions) or two infusion sites $(30.5 \%, 321$ of 1053 infusions). When analyzed by age groups, it appears that younger participants ( $\geq 2$ and $\leq 5$ years of age, $\mathrm{n}=2)$ preferred to use two sites $(86.2 \%, 25$ of 29 infusions). There was a gradual increase in use of four infusion sites as age increased. Older participants (>16 years old) typically used four sites (69.6\%, 525 of 754 infusions). Infusion volume was influenced by the age of the participants. The younger the participants were, the less volume was required to provide the specified $\mathrm{mg} / \mathrm{kg}$ dose of IGSC-C $20 \%$. The mean (SD) duration per infusion for the SC phase was $1.6(0.8) \mathrm{h}$, which is relatively shorter than 2.19 (1.02) $\mathrm{h}$ for the run-in phase and 2.30 (1.04) $\mathrm{h}$ for the IV phase. IGSC-C $20 \%$ was given with the mean (SD) dose of $179(45) \mathrm{mg} / \mathrm{kg} /$ week for a median treatment duration of 24 weeks (mean [SD], 21.6 [6.5] weeks). The median (range) dose was 171 (71-276) mg/kg/week. The total exposure of IGSC-C $20 \%$ was 20.28 subject-years and 1053 infusions (Tables $3 \&$ Supplementary Table 4).

\section{Safety \& tolerability}

About 53 participants received a total of 261 IGIV-C 10\% infusions during the combined run-in + IV phases, and 49 participants received a total of 1053 IGSC-C 20\% infusions during the SC phase (Table 6). The majority of AEs in this study were deemed mild $(63.3 \%, 50 / 79$, run-in + IV; $60.3 \%, 85 / 141$, SC) or moderate $(35.4 \%, 28 / 79$, run-in + IV; 36.2\%, 51/141, SC) in severity. Before any treatment initiation, 22.6\% (12 of 53) of participants experienced $\geq 1 \mathrm{AE}$, mostly related to pre-existing medical conditions. During the run-in $+\mathrm{IV}$ phases, a total of 79 AEs occurred in 62.3\% (33 of 53, 0.303 event per infusion) of participants, and 141 AEs occurred in 83.7\% (41 of 49, 0.134 event per infusion) of the participants during the SC phase - most of which were local infusion site AEs associated with the SC route of administration (Table 7).

The incidences of potentially related AEs were similar between the run-in + IV (0.050 event per infusion) and SC phases (0.058 event per infusion). During the run-in + IV phases, $15.1 \%$ (eight of 53) of participants had 13 potentially infusion-related AEs, including two headaches (3.8\%, two of 53; 0.008 event per infusion) and one each (1.9\%, one of 53; 0.004 event per infusion) of abdominal discomfort, asthma, positive Coombs direct 
Table 5. Summary of subcutaneous administration characteristics (safety population).

\begin{tabular}{|c|c|}
\hline SC Infusions & SC phase $(n=49)$ \\
\hline Total number of SC infusions & 1053 \\
\hline Number of infusion sites used per infusion, mean $\pm S D$ & $3.3 \pm 1.1$ \\
\hline \multicolumn{2}{|l|}{ Number of infusion sites used per infusion, ${ }^{\dagger} \mathrm{n}$ (\% of infusions) } \\
\hline-1 & $30(2.8)$ \\
\hline-2 & $321(30.5)$ \\
\hline-3 & $86(8.2)$ \\
\hline-4 & $592(56.2)$ \\
\hline-6 & $24(2.3)$ \\
\hline \multicolumn{2}{|l|}{ Distribution of SC infusion sites } \\
\hline Total number of participants infused ${ }^{\ddagger}$ & 49 \\
\hline - Abdomen, n (\%) & $46(93.9)$ \\
\hline - Thigh, n (\%) & $19(38.8)$ \\
\hline - Buttocks, n (\%) & $3(6.1)$ \\
\hline - Side(s), n (\%) & $3(6.1)$ \\
\hline - Arm, n (\%) & $1(2.0)$ \\
\hline - Back, n (\%) & $1(2.0)$ \\
\hline - Hip, n (\%) & $1(2.0)$ \\
\hline Total number of SC infusion sites used ${ }^{\S}$ & 3442 \\
\hline - Abdomen, n (\%) & $2513(73.0)$ \\
\hline - Thigh, n (\%) & $746(21.7)$ \\
\hline - Buttocks, n (\%) & $83(2.4)$ \\
\hline -Arm, n (\%) & $46(1.3)$ \\
\hline - Side(s), n (\%) & $32(0.9)$ \\
\hline - Back, n (\%) & $18(0.5)$ \\
\hline - Hip, n (\%) & $4(0.1)$ \\
\hline Dose $(\mathrm{mg} / \mathrm{kg} /$ week), mean $\pm \mathrm{SD}$ & $179 \pm 45$ \\
\hline Duration of treatment period (week), mean $\pm S D$ & $21.6 \pm 6.5$ \\
\hline Volume infused per site per infusion (ml/site), I mean \pm SD & $20.6 \pm 11.5$ \\
\hline Infusion rate per site per infusion ( $\mathrm{ml} /$ hour $/$ site)," mean \pm SD & $16.0 \pm 6.9$ \\
\hline Duration of infusion (h), mean \pm SD & $1.6 \pm 0.8$ \\
\hline By-subject maximum volume infused per site per infusion ( $\mathrm{ml} / \mathrm{site})$, mean \pm SD & $23.5 \pm 20.3$ \\
\hline By-subject maximum infusion rate per site per infusion $(\mathrm{ml} / \mathrm{h} / \mathrm{site})$, mean $\pm \mathrm{SD}$ & $17.5 \pm 8.1$ \\
\hline \multicolumn{2}{|c|}{$\begin{array}{l}\text { †The denominator for the percentages is the total number of } \mathrm{SC} \text { infusions. } \\
\text { ¥SC infusion sites are not mutually exclusive. The denominator for the percentages is the total number of participants infused. } \\
\text { \$The denominator for the percentages is the total number of } \mathrm{SC} \text { infusion sites used. } \\
\text { IVolume infused per site ( } \mathrm{ml} / \mathrm{site})=\text { total volume infused }(\mathrm{ml}) / \text { total number of infusion sites. } \\
\text { \#Infusion rate per site }(\mathrm{ml} / \mathrm{h} / \text { site) }=\text { total infusion rate }(\mathrm{ml} / \mathrm{h}) / \text { total number of infusion sites. } \\
\text { SC: Subcutaneous; } \mathrm{SD}: \text { Standard deviation. }\end{array}$} \\
\hline
\end{tabular}

test, positive Coombs test, cough, granulocytopenia, laryngitis, procedural vomiting, proteinuria, rash pustular and sinusitis. Systemic AEs were low during the SC phase with only one report of headache related to infusions $(2.0 \%$, one of 49; 0.001 event per infusion). All but one of the potentially related AEs in the SC phase were also mild or moderate severity. The one subject, who experienced a severe potentially related AE during the SC phase (on day 20), had polymyalgia rheumatica which was considered unlikely related to study drug and resolved by day 75 .

There were more participants with AEs during or within $72 \mathrm{~h}$ of an infusion in the SC phase $(71.4 \%, 35$ of 49 , 0.087 event per infusion) than in the run-in + IV phases (18.9\%, ten of 53, 0.042 event per infusion). Most were local infusion site AEs associated with the SC route of administration, and the percentage of participants reporting local ISRs declined over time. At the beginning of the SC phase (visit SC\#1), 34.7\% (17 of 49) of participants reported local ISRs. Then at visit SC\#12, 18.2\% (eight of 44) reported local ISRs, and by the end of the SC phase after 24 infusions, only 14.3\% (6 of 42) reported local ISRs. These time-related changes reflect a $>50 \%$ decrease in local ISRs from the beginning of the SC phase as participants continued to receive weekly SC infusions. 
Table 6. Overall summary of adverse events during study phases (safety population).

\begin{tabular}{|c|c|c|c|c|}
\hline \multirow[t]{2}{*}{ Parameters } & \multicolumn{2}{|c|}{ Run-in + IV phases } & \multicolumn{2}{|c|}{ SC phase } \\
\hline & $\begin{array}{l}\text { Patients, } n(\%)^{\dagger} \\
(n=53)\end{array}$ & $\begin{array}{l}\text { Events per infusion, } n \\
\text { (rate) })^{\ddagger} \\
(n=261)\end{array}$ & $\begin{array}{l}\text { Patients, } n(\%)^{\dagger} \\
(n=49)\end{array}$ & $\begin{array}{l}\text { Events per infusion, } n \\
\text { (rate })^{\ddagger} \\
(n=1053)\end{array}$ \\
\hline \multicolumn{5}{|l|}{ Overall summary of adverse events } \\
\hline Any $A E$ & $33(62.3)$ & $79(0.303)$ & $41(83.7)$ & $141(0.134)$ \\
\hline Any potentially related $\mathrm{AE}$ & $8(15.1)$ & $13(0.050)$ & $21(42.9)$ & $61(0.058)$ \\
\hline Any definitely related $A E$ & 0 & 0 & $14(28.6)$ & $42(0.040)$ \\
\hline Any serious adverse event & $1(1.9)^{\S}$ & $2(0.008)$ & $2(4.1) \mathbb{I}$ & $4(0.004)$ \\
\hline AE leading to discontinuation & $1(1.9)^{\#}$ & $2(0.008)$ & $4(8.2)^{\dagger \dagger}$ & $9(0.009)$ \\
\hline Any potentially related $\mathrm{SAE}$ & 0 & 0 & 0 & 0 \\
\hline Death & 0 & 0 & 0 & 0 \\
\hline \multicolumn{5}{|c|}{ 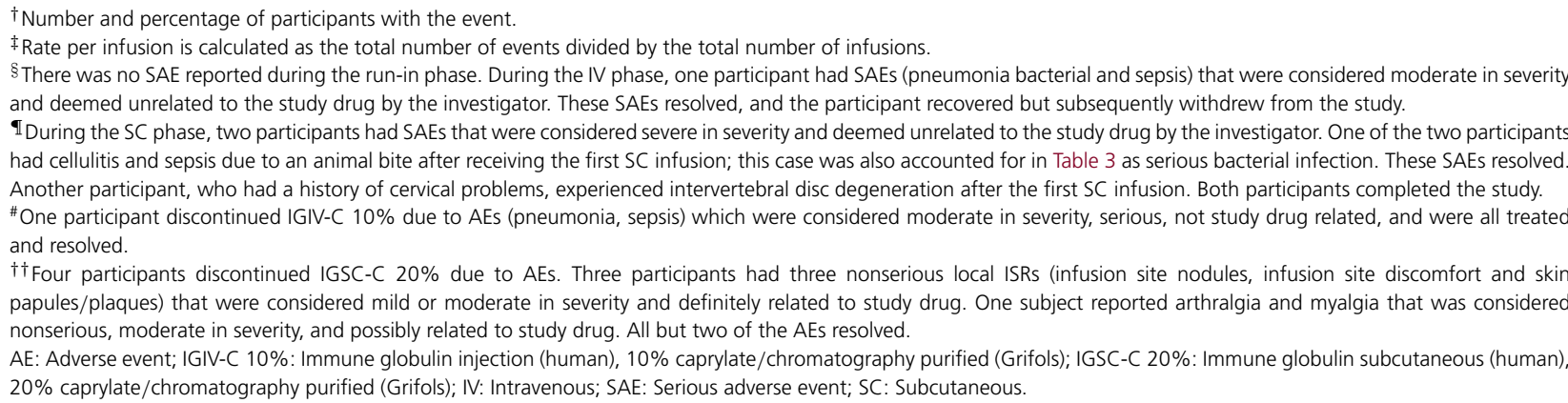 } \\
\hline
\end{tabular}

Table 7. Systemic Adverse events and all local infusion site reactions in $\geq 5 \%$ participants during immune globulin subcutaneous (human), 20\% caprylate/chromatography purified (Grifols) infusions ${ }^{\dagger}$.

\begin{tabular}{|c|c|c|}
\hline Parameters & Patients, $n(\%)^{\ddagger}(n=49)$ & Events per infusion, $n(\text { rate })^{\S}(n=1053)$ \\
\hline \multicolumn{3}{|l|}{ Systemic AEs } \\
\hline - Cough & $3(6.1)$ & $4(0.004)$ \\
\hline - Diarrhea & $3(6.1)$ & $3(0.003)$ \\
\hline \multicolumn{3}{|c|}{ All local infusion site reactions } \\
\hline - Infusion site erythema & $19(38.8)$ & $123(0.117)$ \\
\hline - Infusion site pain & $9(18.4)$ & $32(0.030)$ \\
\hline - Infusion site swelling & $8(16.3)$ & $124(0.118)$ \\
\hline - Infusion site bruising & $8(16.3)$ & $26(0.025)$ \\
\hline - Infusion site nodule & $8(16.3)$ & $13(0.012)$ \\
\hline - Infusion site pruritus & $5(10.2)$ & $28(0.027)$ \\
\hline - Infusion site induration & $4(8.2)$ & $6(0.006)$ \\
\hline - Infusion site scab & $3(6.1)$ & $6(0.006)$ \\
\hline - Infusion site edema & $3(6.1)$ & $5(0.005)$ \\
\hline \multicolumn{3}{|c|}{$\begin{array}{l}\text { †Including all systemic AEs and local infusion site reactions (regardless of whether they met the definition of AEs) that occurred after the first dose of IGSC-C } 20 \% \text { regardless of causality, } \\
\text { excluding infections. } \\
\text { ¥Number and percentage of participants with the event. } \\
\text { § Rate per infusion is calculated as the total number of events divided by the total number of infusions. } \\
\text { AE: Adverse event; IGSC-C 20\%: Immune globulin subcutaneous (human), } 20 \% \text { caprylate/chromatography purified (Grifols); IV: Intravenous; NA: Not applicable; SAE: Serious adverse } \\
\text { event; SC: Subcutaneous. }\end{array}$} \\
\hline
\end{tabular}

Excluding the SBIs discussed above, one participant reported a serious adverse event (SAE) during the SC phase. This SAE was intervertebral disc degeneration, which was considered unrelated to the study drug by the investigator. No SAE occurred in the run-in phase. There was no potentially related SAE, no death, no thromboembolic AEs and no fatigue reported during any study phases. 
In the IV phase, one participant withdrew from the study due to two concurrent SBIs that were considered moderate in severity and not related to study drug. Four participants withdrew from the study during the SC phase. Of those, three participants had three nonserious local ISRs or infusion-related AEs that were considered mild or moderate in severity and definitely related to study drug. One participant reported arthralgia and myalgia that was considered nonserious, moderate in severity and possibly related to study drug. All but two (infusion site nodules) of the AEs resolved. There were no noticeable differences in the AE profiles for the individual age groups (data not shown).

\section{Discussion}

Overall this study had a rigorous design, as only patients with confirmed hypogammaglobulinemia were enrolled to ensure the accuracy of the pharmacokinetic determinations. A key feature was the run-in phase with a $10 \%$ IGIV preparation that had a similar formulation to the $20 \%$ IGSC product. The pharmacokinetic outcomes were comparable with other studies of IGSC $[15,23,24]$. At a dose conversion factor of 1.37 , IGSC-C $20 \%$ provided comparable (noninferior and bioequivalent) overall serum exposure to total $\operatorname{IgG}$ (AUC) relative to the IV dose of IGIV-C $10 \%$ over an equal time interval. Steady-state mean trough total IgG following IGSC-C $20 \%$ administration at 1.37 -times the IV dose averaged 33\% higher than the trough concentrations observed for the IGIV-C 10\% dose. The results indicate the primary $\mathrm{PK}$ end point (steady state $\mathrm{AUC}_{0-7}$ days $)$ is consistent across the three analyses (range $=1.04-1.06$ ), demonstrating the robustness of the analysis results. The lower bound of the $90 \% \mathrm{CI}$ for the GLSM ratio (SC/IV) was above 0.80 , demonstrating noninferiority of IGSC-C $20 \%$ to IGIV-C $10 \%$. In addition, the overall $90 \%$ CI falls within the range of $0.80-1.25$, an accepted criterion for 'bioequivalence' between the two treatments based on regulatory guidelines [25]. The levels of IgG subclasses were consistent with a physiologic distribution pattern (mean values) [26]. Furthermore, mean trough levels among the IgG subclasses displayed similar PK between IGIV-C 10\% and IGSC-C 20\%. These results were expected due to the difference in half-life among the IgG subclasses [27]. Current FDA guidelines require IGSC and IGIV preparations to demonstrate similar IgG AUC, resulting in dosing adjustments ranging from 120 to 153\%. Comparison of IgG trough and AUC between the IGIV and IGSC products tested in this study reveals essentially a linear relationship between formulation and overall bioavailability that is similar to other IGSC products [23,24,28-30].

The rate of SBIs per person per year was low for both SC and IV formulations, although the enrollment numbers were too small to accurately determine efficacy in preventing SBIs. Overall rates of infections of validated infections per person per year were similar between IGSC-C 20\% and IGIV-C 10\%. Furthermore, these rates are similar to reported rates of SBI and other infections among other 20\% IGSC products [15,24,31-35,24].

IGSC-C $20 \%$ administration was not associated with any reports of serious systemic or local ISRs; the majority of nonserious local ISRs observed were mild to moderate, with an incidence that decreased over time (repeated SC infusions). Infusion-related systemic AEs (i.e., headache) were low, $<0.001$ per infusion. The number, site locations and infusion volume per site used by the participants varied among the cohort as both children and adults were included in the study. Overall, most participants used abdomen or thighs as site locations, with the average volume per site near $20 \mathrm{ml}$, but with a high degree of individual variation. The average infusion rate at just over an hour allows for convenient infusions in the home care setting.

It is important to keep in mind that participants received many more SC infusions ( $\mathrm{n}=1053)$ than IV infusions $(n=261)$, and this difference in the number of infusions for the two treatment groups contributed to the lower calculated rate of AEs per infusion for SC administration (0.134 event per infusion) relative to IV administration (0.303 event per infusion). On the other hand, it could also have contributed to the higher rate of AEs during or within $72 \mathrm{~h}$ of an infusion in the SC phase.

SC administration of IGSC-C 20\% maintained equivalent exposure to IGIV-C 10\% administered IV, with higher mean trough values and lower fluctuations in IgG concentrations. Administration in the home care setting was tolerated in both children and adults enrolled in the study. Before the approval of this study product in July 2019, there were two FDA-approved IGSC-C 20\% products available in the USA: Hizentra ${ }^{\circledR} 20 \%$ [32], a proline-stabilized IGSC (CSL Behring, approved 2010) and Cuvitru ${ }^{\circledR} 20 \%$ [10], a glycine-stabilized IGSC (Baxalta, approved 2016). Overall, the rates of infections and SBIs in participants given IGSC-C $20 \%$ as IgG replacement therapy for immune prophylaxis were low and comparable with other subcutaneous IgG products.

This study was designed in accordance with FDA guidelines in terms of study design and end points and included multiple centers. As such the limitations in the study were mainly with regard to the youngest age category 
(2-5 years), wherein two patients enrolled and one completed study. Thus, the results for very young children were limited within the confines of this trial alone.

Another Phase III study (NCT02806986) that was designed according to European Medicines Agency guidelines, employing a 1:1 DAF, recently completed clinical phase in Europe. We anticipate that results from this study will further guide physicians on how to treat PI using IGSC-C $20 \%$.

In conclusion, subcutaneous administration of IGSC-C $20 \%$ was safe and well tolerated with a safety profile comparable to IV administration of IGIV-C $10 \%$.

\section{Summary points}

- Immune globulin subcutaneous, human - klhw (IGSC-C 20\%) is a $20 \%$ immunoglobulin G (IgG) solution for subcutaneous injection that is US FDA-approved for the treatment of primary humoral immunodeficiency (PI) in patients 2 years of age and older.

- This study was a prospective, multicenter, open-label and Phase III clinical trial (NCT02604810) evaluating the pharmacokinetics, safety and tolerability of IGSC-C $20 \%$, compared with immune globulin injection (human), $10 \%$ caprylate/chromatography purified (IGIV-C $10 \%$ ), in patients with $\mathrm{PI}$.

- Subcutaneous (SC) administration of IGSC-C $20 \%$ maintained equivalent exposure to IGIV-C $10 \%$ administered IV, with higher mean trough values (SC:IV of 1.333) and lower fluctuations in IgG concentrations.

- The average (standard deviation) of the steady-state mean trough concentrations of total IgG over all participants was approximately 957 (242) mg/dl for IGIV-C 10\% and approximately 1245 (272) mg/dl for IGSC-C $20 \%$.

- IGSC-C $20 \%$ was safe and well tolerated with a safety profile comparable to IV administration of IGIV-C $10 \%$.

- SBIs per person per year were 0.120 (95\% Cl: 0.051-0.232; upper $99 \%$ confidence limit: 0.259$)$ in the IGIV-C $10 \%$ Run-in + IV phase and 0.049 (95\% Cl, 0.020-0.098; upper $99 \%$ confidence limit: 0.110$)$ on IGSC-C $20 \%$ treatment.

- The rate of infections per person per year was comparable between IGIV-C $10 \%$ treatment $(0.658[95 \% \mathrm{Cl}$, $0.378-1.051])$ and IGSC-C $20 \%$ treatment $(0.493$ [95\% Cl, 0.273-0.809]).

- Serum trough concentrations of antibody for H. influenzae, C. tetani, and S. pneumoniae increased after switching from IV to SC phases and were generally consistent across all age groups. The trough concentrations of measles antibody were comparable between the IV and SC phases and were protective.

- A total of 79 adverse events (AEs) occurred in $62.3 \%$ (33 of 53, 0.303 event per infusion) of participants during IGIV-C $10 \%$ treatment, and 141 AEs occurred in $83.7 \%$ (41 of $49,0.134$ event per infusion) of the participants during IGSC-C $20 \%$ treatment-most of which were local infusion site AEs associated with the SC route of administration.

- The majority of AEs ( $98.7 \%$ for IGIV-C $10 \% ; 96.5 \%$ for SC) were mild or moderate in severity.

- There was no potentially related serious AEs or death reported in this study.

\section{Author contributions}

All authors significantly contributed to trial execution and acquisition of data, reviewed and contributed to manuscript finalization. In addition, JW Sleasman critically reviewed, significantly revised initial drafts of the manuscript and made substantial contribution to the interpretation of the data. E Mondou and KL Courtney made substantial contribution to conception and design, and the interpretation of the data. E Mondou was the medical director for this study, and JL performed statistical analyses.

\section{Supplementary data}

To view the supplementary data that accompany this paper please visit the journal website at: www.futurescience.com/doi/suppl/10.2217/imt-2019-0159

\section{Acknowledgments}

The authors thank TM Nguyen-Cao, CMPP of Grifols for medical writing services under the direction of the authors. We also thank the following investigators who gave us permission to acknowledge their participation in this trial: AL Darter, MD of Oklahoma Institute of Allergy \& Asthma Clinical Research, Oklahoma City, OK, USA; M Ballow of Johns Hopkins All Children's Hospital St Petersburg, FL, USA; D Cameron and J Cowan of The Ottawa Hospital Research Institute, Ottawa, Ontario, Canada; MI Garcia Lloret of UCLA School of Medicine, Los Angeles, CA, USA; E Haddad of CHU Ste-Justine, Montreal, Quebec, Canada; S Kumar of VCU Health System - Children's Hospital of Richmond, Richmond, VA, USA; J Moy of Rush University Medical Center, Chicago, IL, USA; Pavadee Poowuttikul of Children's Hospital of Michigan, Detroit, MI, USA; R Shapiro of Midwest Immunology Clinic, Plymouth, MN, USA; R Tachdjian AIRE Medical of Los Angeles, Santa Monica, CA, USA. The authors appreciate the dedication of site staff and the commitment of enrolled participants. After the conduct of this study and development of this manuscript, Kecia Courtney has changed affiliation to Biogen, 5000 Davis Drive, Research Triangle Park, NC 27709 (kecia.courtney@gmail.com). 
Financial \& competing interests disclosure

This study was supported by Grifols, Research Triangle Park, NC, USA. Grifols is a manufacturer of IGSG 20\% and IGIV-C 10\%. The opinions in this manuscript are those of the authors. J Sleasman, I Hussain and J Harris served as principal investigators in this study sponsored by Grifols; they have no other conflicts of interest related to this publication. M Stein was a speaker and investigator for Shire; and an investigator for BPL, Green Cross, Grifols, Therapure, Kedrion and Prometic. W Lumry has consultant agreements with ALK, Adverum, BioCryst, CSL Behring, Optinose, Shire; participates in speakers' bureau of ALK, Adverum, BioCryst, CSL Behring, Optinose, Shire; and receives grants/research support from Attenua, BioCryst, Biotest, CSL Behring, Genentech, Grifols, Glenmark, Green Cross, GSK, Kedrion, Menlo, Novartis, Pearl, Therapure, Sanofi, Shire and Watson. J Wedner is on the advisory board for Takeda Pharmaceuticals and CSL-Behring Pharmaceuticals and receives research support from Grifols, Takeda and CSL Behring; he has no other conflicts of interest related to this publication. E Mondou and J Lin are employees of Grifols. K Courtney was an employee of Grifols during the conduct of this study and the development of this manuscript. The authors have no other relevant affiliations or financial involvement with any organization or entity with a financial interest in or financial conflict with the subject matter or materials discussed in the manuscript apart from those disclosed.

TM Nguyen-Cao CMPP of Grifols provided medical writing services under the direction of the authors.

Ethical conduct of research

This study was conducted in full conformance with local laws and regulations and the Declaration of Helsinki. The protocol was approved by the Institutional Review Board/Ethics Committee of all participating sites, and informed consent was obtained from each patient and/or patients' parents or legal guardian prior to screening.

Data sharing statement

The authors certify that this manuscript reports the secondary analysis of clinical trial data that have been shared with them, and that the use of this shared data is in accordance with the terms (if any) agreed upon their receipt. The source of this data is: NCT02604810. Data reported in this manuscript are available within the article and its supplementary materials. Additional studylevel data from the Safety and Pharmacokinetics of IGSC-C 20\% in participants with primary immunodeficiency (NCT02604810) will be available at ClinicalTrials.gov.

\section{Open access}

This work is licensed under the Attribution-NonCommercial-NoDerivatives 4.0 Unported License. To view a copy of this license, visit http://creativecommons.org/licenses/by-nc-nd/4.0/

\section{References}

Papers of special note have been highlighted as: $\bullet$ of interest; $\bullet \bullet$ of considerable interest

1. Picard C, Bobby Gaspar H, Al-Herz W et al. International Union of Immunological Societies: 2017 primary immunodeficiency diseases committee report on inborn errors of immunity. J. Clin. Immunol. 38(1), 96-128 (2018).

- Demonstrates the expanding number of causes of primary immune deficiency.

2. Bousfiha A, Jeddane L, Picard C et al. The 2017 IUIS phenotypic classification for primary immunodeficiencies. J. Clin. Immunol. 38(1), 129-143 (2018).

3. Bonilla FA, Khan DA, Ballas ZK et al. Practice parameter for the diagnosis and management of primary immunodeficiency. J. Allergy Clin. Immunol. 136(5), 1186-1205 e1181-1178 (2015).

- Summarizes the American Academy of Allergy Asthma \& Immunology recommendations for the infusions of intravenous and subcutaneous immunoglobulin.

4. Boyle J, Buckley RH. Population prevalence of diagnosed primary immunodeficiency diseases in the United States. Pharmaceuticals Policy Law 10, 99-108 (2008).

5. Kobrynski L, Powell RW, Bowen S. Prevalence and morbidity of primary immunodeficiency diseases, United States 2001-2007. J. Clin. Immunol. 34(8), 954-961 (2014).

6. Rh B, Ri S. The use of intravenous immune globulin in immunodeficiency diseases. N. Engl. J. Med. 325(2), 110-117 (1991).

7. Sacher RA, Panel IA. Intravenous immunoglobulin consensus statement. J. Allergy Clin. Immunol. 108(Suppl. 4), S139-S146 (2001).

8. Perez EE, Orange JS, Bonilla F et al. Update on the use of immunoglobulin in human disease: a review of evidence. J. Allergy Clin. Immunol. 139(3S), S1-S46 (2017).

9. CUTAQUIG ${ }^{\circledR}$ (immune globulin subcutaneous (human) - hipp), 16.5\% [package insert]. Octapharma USA Inc, Hoboken, NJ, USA (2018).

10. CUVITRU, immune globulin subcutaneous (human), 20\% solution [package insert]. Baxalta US Inc, Lexington, MA, USA (2018). 
11. HIZENTRA, immune globulin subcutaneous (human), 20\% liquid [package inserts]. CSL Behring LLC, Kankakee, IL, USA (2018).

12. Jolles S, Sleasman JW. Subcutaneous immunoglobulin replacement therapy with Hizentra, the first $20 \%$ SCIG preparation: a practical approach. Adv. Ther. 28(7), 521-533 (2011).

- This is summary of the use of a $20 \%$ proline stabilized subcutaneous immunoglobulin.

13. XEMBIFY ${ }^{\circledR}$ (immune globulin subcutaneous, human - klhw) 20\% solution [package insert]. Grifols Therapeutics LLC, Research Triangle Park, NC, USA (2019).

14. GAMUNEX ${ }^{\circledR}-\mathrm{C}$ [immune globulin injection (human), $10 \%$ caprylate/chromatography purified] [package insert]. Grifols Therapeutics LLC, Research Triangle Park, NC, USA (2018).

15. Jolles S, Bernatowska E, De Gracia J et al. Efficacy and safety of Hizentra((R)) in patients with primary immunodeficiency after a dose-equivalent switch from intravenous or subcutaneous replacement therapy. Clin. Immunol. 141(1), 90-102 (2011).

16. HYQVIA, immune globulin subcutaneous (human), 20\% liquid [package insert]. Baxalta US Inc, Lexington, MA, USA (2019).

17. Orange JS, Hossny EM, Weiler CR et al. Use of intravenous immunoglobulin in human disease: a review of evidence by members of the Primary Immunodeficiency Committee of the American Academy of Allergy, Asthma and Immunology. J. Allergy Clin. Immunol. 117(Suppl. 4), S525-S553 (2006).

18. Guidance for industry: safety, efficacy, and pharmacokinetic studies to support marketing of immune globulin intravenous (human) as replacement therapy for primary humoral immunodeficiency. Docket Number:

2005D-0438 (2008). https://www.fda.gov/regulatory-information/search-fda-guidance-documents/safety-efficacy-and-pharmacokine tic-studies-support-marketing-immune-globulin-intravenous-human

19. Orange JS, Grossman WJ, Navickis RJ, Wilkes MM. Impact of trough IgG on pneumonia incidence in primary immunodeficiency: A meta-analysis of clinical studies. Clin Immunol 137(1), 21-30 (2010).

20. Fried AJ, Bonilla FA. Pathogenesis, diagnosis, and management of primary antibody deficiencies and infections. Clin. Microbiol. Rev. 22(3), 396-414 (2009).

21. Immune Globulin Potency in the 21st Century CBER Nov 8-9, 2017 Rockville, MD. https://www.fda.gov/vaccines-blood-biologics/w orkshops-meetings-conferences-biologics/immune-globulin-potency-21st-century-public-workshop-11072017-11072017

22. Young MK, Bertolini J, Kotharu P, Maher D, Cripps AW. Do Australian immunoglobulin products meet international measles antibody titer standards? Hum. Vaccin. Immunother. 13(3), 607-612 (2017).

23. Berger M, Jolles S, Orange JS, Sleasman JW. Bioavailability of IgG administered by the subcutaneous route. J. Clin. Immunol. 33(5), 984-990 (2013).

- Discusses the dosing and bioavailability of transition from intravenous to subcutaneous immunoglobulin therapy.

24. Suez D, Stein M, Gupta S et al. Efficacy, safety, and pharmacokinetics of a novel human immune globulin subcutaneous, $20 \%$ in patients with primary immunodeficiency diseases in North America. J. Clin. Immunol. 36(7), 700-712 (2016).

- This is a study of a $\mathbf{2 0} \%$ glycine-stabalized subcutaneous immunoglobulin.

25. U.S. Department of Health and Human Services, Food and Drug Administration, Center for Drug Evaluation and Research (CDER). Guidance for Industry - statistical approaches to establishing bioequivalence. 1-45 (2001). https:

//www.fda.gov/regulatory-information/search-fda-guidance-documents/statistical-approaches-establishing-bioequivalence

- This publication reviews the FDA guidelines for establishing Bioequivalence.

26. Jefferis R, Kumararatne DS. Selective IgG subclass deficiency: quantification and clinical relevance. Clin. Exp. Immunol. 81(3), 357-367 (1990).

27. Vidarsson G, Dekkers G, Rispens T. IgG subclasses and allotypes: from structure to effector functions. Front. Immunol. 5, 520 (2014).

28. Wasserman RL, Irani AM, Tracy J et al. Pharmacokinetics and safety of subcutaneous immune globulin (human), 10\% caprylate/chromatography purified in patients with primary immunodeficiency disease. Clin. Exp. Immunol. 161(3), 518-526 (2010).

-. Demonstrates the safety and efficacy of Gamunex-C 10\%, in the treatment of adults with primary immunodeficiency.

29. Wasserman RL, Melamed I, Nelson RP Jr et al. Pharmacokinetics of subcutaneous IgPro20 in patients with primary immunodeficiency. Clin. Pharmacokinet. 50(6), 405-414 (2011).

30. Wasserman RL, Melamed I, Kobrynski L et al. Efficacy, safety, and pharmacokinetics of a $10 \%$ liquid immune globulin preparation (GAMMAGARD LIQUID, 10\%) administered subcutaneously in subjects with primary immunodeficiency disease. J. Clin. Immunol. 31(3), 323-331 (2011).

31. Niebur HB, Duff CM, Shear GF et al. Efficacy and tolerability of $16 \%$ subcutaneous immunoglobulin compared with $20 \%$ subcutaneous immunoglobulin in primary antibody deficiency. Clin. Exp. Immunol. 181(3), 441-450 (2015).

32. Araga S, Leboeuf RD, Blalock JE. Prevention of experimental autoimmune myasthenia gravis by manipulation of the immune network with a complementary peptide for the acetylcholine receptor. Proc. Natl Acad. Sci. USA 90(18), 8747-8751 (1993).

33. GAMMAKED ${ }^{\mathrm{TM}}$, [immune globulin injection (human), 10\% caprylate/chromatography purified] [package insert]. Kedrion Biopharma Inc, Fort Lee, NJ, USA (2017). 
34. Heimall J, Chen J, Church JA, Griffin R, Melamed I, Kleiner GI. Pharmacokinetics, safety, and tolerability of subcutaneous immune globulin injection (human), $10 \%$ caprylate/chromatography purified (GAMUNEX ${ }^{\circledR}-\mathrm{C}$ ) in pediatric patients with primary immunodeficiency disease. J. Clin. Immunol. 36(6), 600-609 (2016).

- Demonstrates the safety and efficacy of Gamunex-C $10 \%$ in the treatment of children with primary immunodeficiency.

35. Kanegane H, Imai K, Yamada M et al. Efficacy and safety of IgPro20, a subcutaneous immunoglobulin, in Japanese patients with primary immunodeficiency diseases. J. Clin. Immunol. 34(2), 204-211 (2014).

36. Hagan JB, Fasano MB, Spector $S$ et al. Efficacy and safety of a new $20 \%$ immunoglobulin preparation for subcutaneous administration, IgPro20, in patients with primary immunodeficiency. J. Clin. Immunol. 30(5), 734-745 (2010). 